\title{
DIVERSITY PROFILING OF ASSOCIATED BACTERIA FROM THE SOILS OF STRESS TOLERANT PLANTS FROM SEACOAST OF JEDDAH, SAUDI ARABIA
}

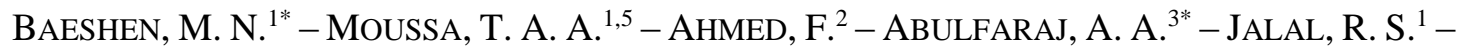 \\ MAJEED, M. A. ${ }^{1}-$ BAESHEN, N. A. ${ }^{4}-$ HUELSENBECK, J. P. ${ }^{1,6}$ \\ ${ }^{1}$ Department of Biology, College of Science, University of Jeddah, Jeddah 21598, Saudi Arabia \\ ${ }^{2}$ Department of Biochemistry, College of Science, University of Jeddah, Jeddah 21598, Saudi \\ Arabia
}

${ }^{3}$ Department of Biological Sciences, Science and Arts College, Rabigh Campus, King Abdulaziz University (KAU), Jeddah, Saudi Arabia

${ }^{4}$ Department of Biology, Faculty of Science, King Abdulaziz University, Jeddah 21598, Saudi Arabia

${ }^{5}$ Botany and Microbiology Department, Faculty of Science, Cairo University, Giza 12613, Egypt

${ }^{6}$ Department of Integrative Biology, University of California, Berkeley, USA

*Corresponding authors

e-mail:mnbaeshen@uj.edu.sa; aaabulfaraj@kau.edu

(Received $28^{\text {th }}$ Jul 2020; accepted $7^{\text {th }}$ Oct 2020)

\begin{abstract}
Soils associated with halophytic plants naturally contain a number of ubiquitous microbial communities facing limited nutrients and harsh environmental conditions including salinity and drought. In the present study, metagenomic sequencing of 16S rRNA was used to analyze and classify bacterial communities of the soil associated with halophytic plants Halopeplis perfoliata and Zygophyllum album collected from various soil samples located in the seacoast of Jeddah province, Saudi Arabia. Analysis of the 16S rRNA sequences at the taxonomic phylum-level revealed that bacterial communities in the soil samples belonged to nineteen phyla, and the most abundant were highlighted for further analysis. Results indicated that the most common phyla were Proteobacteria, Actinobacteria, Firmicutes, Bacteroidetes, Deinococcus-Thermus, Gemmatimonadetes, and an unclassified phyla. At the taxonomic genus-level, the most abundant ones were highlighted for further analysis which include Marinicauda, Altererythrobacter, Maricurvus, Marinobacter, Porticoccus, Salicola, and three unclassified genera were found belonging to Proteobacteria. Actinopolyspora, Geodermatophilus, Propionibacterium, Euzebya and four unclassified ones were found associated with Actinobacteria. Bacillus, Staphylococcus, Paenibacillus, Lactobacillus, Streptococcus, Symbiobacterium, and one unclassified genus were found in Firmicutes. Salegentibacter, Haliscomenobacter, and one unclassified genus of Bacteroidetes. Truepera was found in DeinococcusThermus and Gemmatimonas was found in Gemmatimonadetes. Studying taxonomic, phylogenetic, and functional diversity of soil microbiome will provide a better understanding for novel candidates that can be selected as biological agents to improve agricultural and industrial practices.
\end{abstract}

Keywords: microbiome, PGPB, metagenomics, Halopeplis perfoliate, Zygophyllum album

\section{Introduction}

Soil is probably the most complex and dynamic natural ecosystem providing a favorable environment for the growth and production of huge number of 
microorganisms depending on the soil $\mathrm{pH}$, chemical and physical properties (del Carmen Orozco-Mosqueda et al., 2018), and geographic positions (Bui, 2013). These microorganisms play a critical role in regulating plant life cycle and health through biomass decomposition, soil fertility, and cycling of nitrogen, carbon, and other nutrients. Thousands of bacterial, archaeal, and eukaryotic taxa can be found in every gram of soil and this taxonomic diversity is reflected by the diversity of their biological compositions that effect their functions. Moreover, these heterogenous microbial communities can live either as a free-living or symbiotic and their influence can vary from pathogenic to beneficial, or mutualistic (Chaparro et al., 2012; Fierer et al., 2012).

Plant growth-promoting bacteria (PGPB) are a collection of unrelated bacteria that are found in symbiotic relations with plants and have been adapted as a sustainable alternative for crop production. PGPB can be found in the rhizosphere, epiphytes by attaching the surface of plants roots or leaves, or inside the plant tissues as endophytic bacteria (de Zelicourt et al., 2013; Timmusk et al., 2017). Soil salinity can significantly affect the growth of many plants (Waśkiewicz et al., 2013). However, PGPB promote the growth of plants under harsh environmental conditions including draught, high salinity, and temperature (Majeed et al., 2020). Microorganisms live in a relationship with plants are able to stimulate plant growth by directly obtaining nutrients (nitrogen, iron, phosphate) or regulating the hormone levels including auxins and ethylene, and also by indirectly preventing pathogens from attacking plants (Glick, 2012).

Halopeplis perfoliata plants are halophytic and desert plants that are mostly found in association with symbiotic bacteria in order to stand harsh environmental conditions (Etesami and Beattie, 2018). They can withstand and grow in dry seasons and are mainly found in arid and semi-arid regions and wetlands with high salinity (Etesami and Beattie, 2018; Majeed et al., 2020). Moreover, H. perfoliata have various ecological and industrial benefits including soap and glass industry (Rasool et al., 2017; Zreik, 1990; Al-Oudat and Qadir, 2011). Zygophyllum album is another example of halophytic plants that live in the same community of $H$. perfoliate. It was known as wild desert medicinal plant that was used in folkloric medicine as a diuretic (Mnafgui et al., 2012; Tigrine-Kordjani et al., 2011), local anesthetic (El Ghoul et al., 2011), and for treating various disorders including diabetes mellitus, rheumatism, gout, asthma (Tigrine-Kordjani et al., 2011).

Numerous studies have used the metagenomics approaches in order to understand the soil microbial communities of different ecosystems from different soils collected from cold and hot deserts, forests, grasslands and tundra (Baeshen, 2017). However, very few of them have focused on microbial communities of soils associated with halophytes. It is important to understand how soil microbiome interacts and promotes halophytes to grow and sustain under abiotic stress conditions, and how these halophytes respond to these diverse microbial communities. Such interaction will reveal a huge diversity of microorganisms that are able to foster the growth of diverse crop plants under various biotic and abiotic stresses and can be used as biological agents in numerous industrial and medical applications (Majeed et al., 2020).

The work presented in this study was aimed to discover the microbial diversity found in soils associated with halophytes. We use the metagenomic approaches in order to discover novel promising prokaryotic candidates that can be used as plant growth promoting bacteria and biomolecules of industrial importance. 


\section{Materials and methods}

\section{Sample collection}

The study area was located in the seacoast of Jeddah (Particularly from the Southern Corniche), Saudi Arabia with latitude: 21.13'08.3" N and longitude: 39.10'29.7" E and altitude: $3 \mathrm{~m}$ above sea level. The climate in the Jeddah region is classified as hot, arid, and sandy with a lower amount of rainfall and an average temperature in January on the day ranges between $26-33{ }^{\circ} \mathrm{C}$. Despite all these characteristics, the halophytic plant Halopeplis perfoliata and the Zygophyllum album as a member of $H$. perfoliata communities grow significantly in this region.

Sampling was carried out on the $9^{\text {th }}$ of January 2019 at noon and the temperature was $35^{\circ} \mathrm{C}$. A total of four soil samples associated with two halophytic desert plants namely Halopeplis perfoliata and Zygophyllum album were collected. Two samples from the rhizosphere of each plant with a depth of $10 \mathrm{~cm}$ beneath the first layer and two samples from the crust soil samples associated with Halopeplis perfoliata and Zygophyllum album. in addition, one plant free soil sample was collected from a nearby area that has no plant growth and was used as a control. An amount of $10 \mathrm{~g}$ soil was collected from each sample and were immediately kept in dry ice and store in $-80{ }^{\circ} \mathrm{C}$ until further analysis. Samples codes were as the following; control sample (L3. S3 Control), Halopeplis perfoliata rhizosphere sample (L1.S1.R), Halopeplis perfoliata crust sample (L1.S1.C), Zygophyllum album rhizosphere sample (R3), and Zygophyllum album crust sample (C3).

Identifications of theses salt tolerance plants of the study was carried by our team member, Professor Nabih A. Baeshen as compared to the collection of the preserved specimens in the herbarium of the Department of Biology, Faculty of Science, King Abdulaziz University (Batanouny and Baeshin, 1982, 1983; Sejiny et al., 1980; Zaki et al., 1980).

\section{Genomic DNA isolation, PCR amplification, and $16 \mathrm{~S}$ rRNA gene sequencing}

Soil samples were shipped to Macrogen Inc. Company (Seoul, South Korea) and genomic DNA was extracted from the soil samples. DNA purity and quantification were evaluated using the Picogreen (Invitrogen, cat. \#P7589) fluorescence-based quantification method.

Bacterial V3-V4 16S rRNA gene regions fragments was amplified by PCR with the universal primers (Bakt_341F: CCTACGGNGGCWGCAG) and (Bakt_805R: GACTACHVGGGTATCTAATCC). The PCR amplification program was performed using an initial denaturation at $94^{\circ} \mathrm{C}$ for $5 \mathrm{~min}$, followed by 30 cycles of denaturation at $94{ }^{\circ} \mathrm{C}$ for $30 \mathrm{~s}$, annealing at $57^{\circ} \mathrm{C}$ for $40 \mathrm{~s}$, and extension at $72{ }^{\circ} \mathrm{C}$ for $1.30 \mathrm{~s}$ with a final elongation at $72{ }^{\circ} \mathrm{C}$ for $10 \mathrm{~min}$ (Rawat and Joshi, 2019).

Purified amplicons were used for library construction and deep sequencing on an Illumina SBS technology to recover 300 bp pair-end reads of the V3 and V4 regions.

\section{$16 S$ dataset processing and statistical analysis}

Raw sequencing data was analyzed using Quantitative Insights into Microbial Ecology (QIIME) software (http://qiime.org), which is a bioinformatics open-source tool used in performing microbiome analysis from raw DNA sequencing data that is generated on the Illumina or other sequencing programs. Furthermore, QIIME offers 
quality pretreatment of raw reads, OTU picking, taxonomic assignment, and phylogenetic reconstruction, and diversity analyses and graphical displays (Caporaso et al., 2010).

V3-V4 16S rRNA sequence reads were filtered and trimmed using the CD-HIT-OTU software (http://weizhongli-lab.org/cd-hit-otu/). The FLASH software (http://ccb.jhu.edu/software/FLASH/) was used for merging paired-end reads from nextgeneration sequencing experiments to eliminate the low-quality sequences. Operational taxonomic units (OTUs) were used to linked and classified unique sequence set with a cutoff of 97\% identity. The Ribosomal Database Project (RDP) Classifier was used for taxonomic composition.

Alpha diversity was assessed by Chao1, which was estimated based on the report that came from Macrogen (Chao and Bunge, 2002). Shannon and Simpson indices that were estimated by Mothur software package (http://www.mothur.org) to analyze the complexity of species. Drawing rarefaction curve was based on calculating OTU numbers of the extracted tags and detecting the maximum depth allowable to retain all samples. Beta diversity was detected by calculating the weighted and unweighted UniFrac distances and plotted through principal coordinate analysis (PCoA). UniFrac uses the system evolution information to compare bacterial communities' species between samples. The highest abundance of each genus was selected and genus level phylogenetic tree was drawn by Interactive Tree of Life (ITOL) (https://itol.embl.de).

\section{Results}

\section{S rRNA statistical analysis}

In the present study, metagenomic approach was used a powerful tool to investigate the microbial community structure and diversity of five different soil samples associated with Halopeplis perfoliata and Zygophyllum album. Illumina SBS was used in analyzing the soil different samples based on the 16S rRNA.

The percentage of the read quality of the five soil different samples is shown in Figure A1. Total number of sequences reads and the results of the assembly for the five samples were carried out from FLASH software and is shown in Figure A2 and Table 1, respectively. Data showed a total of clean sequences reads 546,713 with the highest value of 126,158 found in $H$. perfoliata crust sample and the lowest 88,446 value found in the control sample.

Table 1. Result of assembly of the five soil samples

\begin{tabular}{c|c|c|c|c|c}
\hline \multicolumn{7}{c}{ FLASH Software } \\
\hline Sample name & Total bases & Read count & GC $(\%)$ & Q20 $(\%)$ & Q30 $(\%)$ \\
\hline L3S3.Control & $39,889,093$ & 88,446 & 57.84 & 98.11 & 93.32 \\
L1S1R & $46,175,526$ & 102,358 & 56.67 & 98.19 & 93.67 \\
L3S1C & $57,623,348$ & 126,158 & 55.03 & 98.24 & 93.91 \\
R3 & $55,897,213$ & 124,341 & 57.98 & 98.1 & 93.18 \\
C3 & $47,494,924$ & 105,410 & 57.52 & 98.34 & 94.08 \\
Total number & $247,080,104$ & 546,713 & & & \\
\hline
\end{tabular}

Total bases: The total number of bases in reads identified. Read count: The total number of sequence reads. GC (\%): the GC percentage in sequence reads. Q20 (\%): the percentage of bases in which the phred score is above 20. Q30 (\%): The percentage of bases in which the phred score is above 30 


\section{Operational taxonomic unit (OTU) analysis}

CD-HIT-OTU program and rDnaTools were used to filter the sequences from any contamination. Results of clustering of the five soil samples which were assigned to the OTU is shown in (Fig. 1). The highest value of number of OTUs was 333 belongs to $H$. perfoliata crust sample while the lowest was 108 belongs to the control sample.

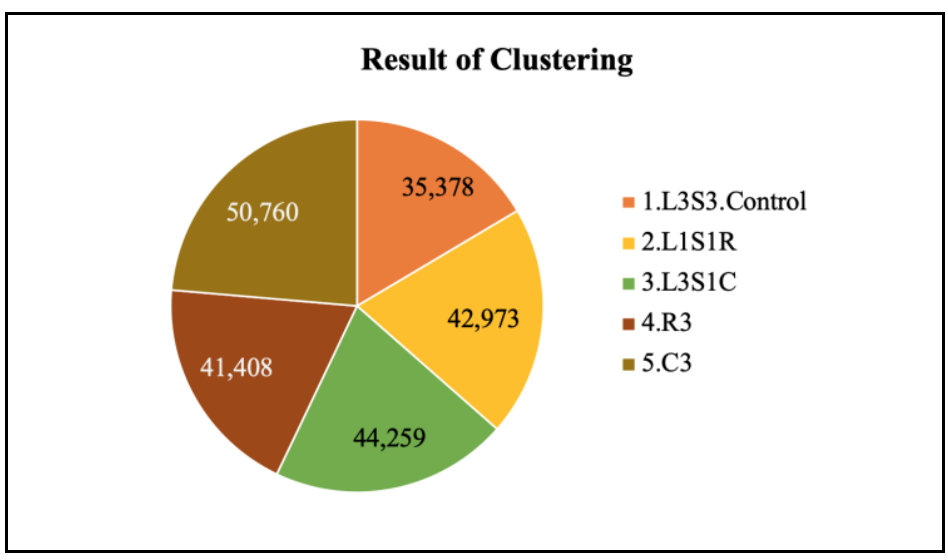

Figure 1. Result of clustering. (L3. S3 Control): Control sample; (L1.S1.R): H. perfoliata rhizosphere sample; (L1.S1.C): H. perfoliata crust sample; (R3) Z. album rhizosphere sample;

(C3) Zygophyllum album crust sample

\section{Community richness and diversity}

Alpha diversity was applied to study the complexity of species through several indices; A. Chaol value describes richness estimates for an OTU definition. B. Shannon value describes the species diversity of the community that affected by both species' richness, and species evenness. C. Inverse Simpson value which represents the probability that two randomly selected individuals in the habitat will belong to the same species. Table 2 shows the results of the OTUs and the alpha diversity metrics (Chao1, Shannon, Simpson) on each sample. The number of OTUs on each sample is shown in Figure 2. Different curves based on observed Shannon value and Inversed Simpson value is shown in Figure 3 and alfa rarefaction curve observed is shown in Figure 4.

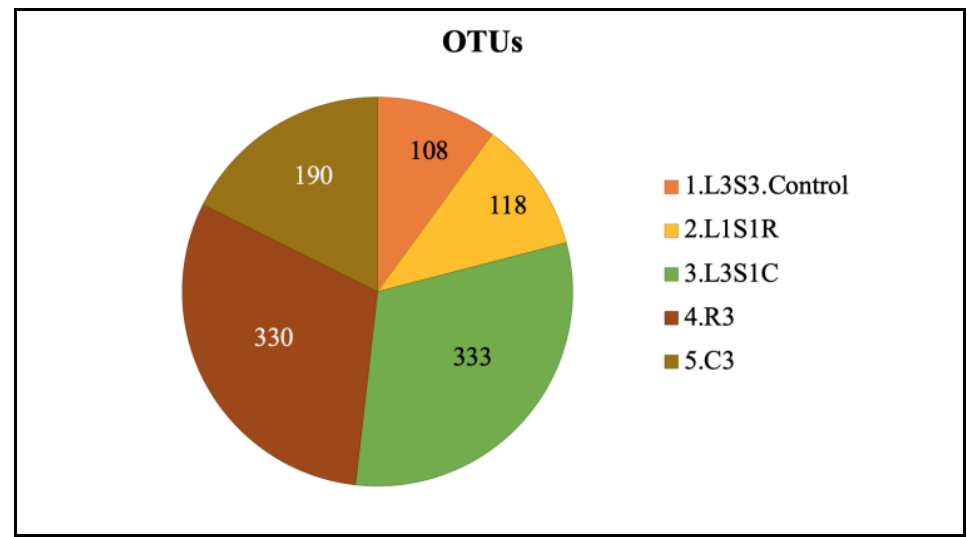

Figure 2. The number of OTUs generated for each sample. (L3. S3 Control): Control sample; (L1.S1.R): H. perfoliata rhizosphere sample; (L1.S1.C): H. perfoliata crust sample; (R3) Z. album rhizosphere sample; (C3) Zygophyllum album crust sample 


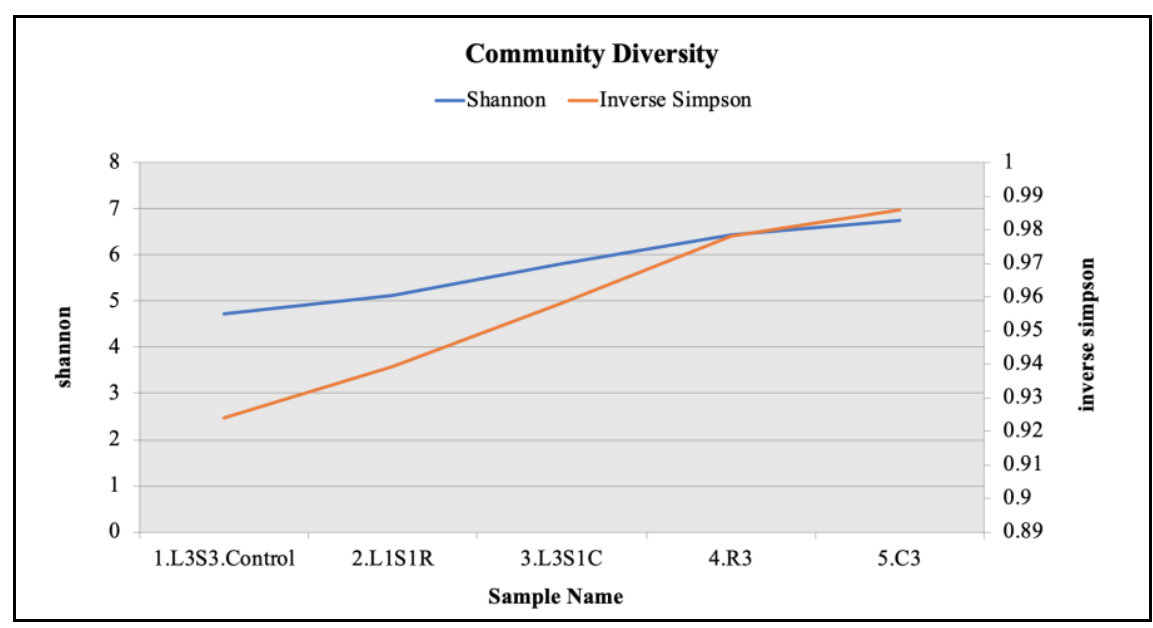

Figure 3. Different curve based on observed Shannon value and inversed Simpson value. (L3. S3 Control): Control sample; (L1.S1.R): H. perfoliata rhizosphere sample; (L1.S1.C): H. perfoliata crust sample; (R3) Z. album rhizosphere sample; (C3) Zygophyllum album crust sample

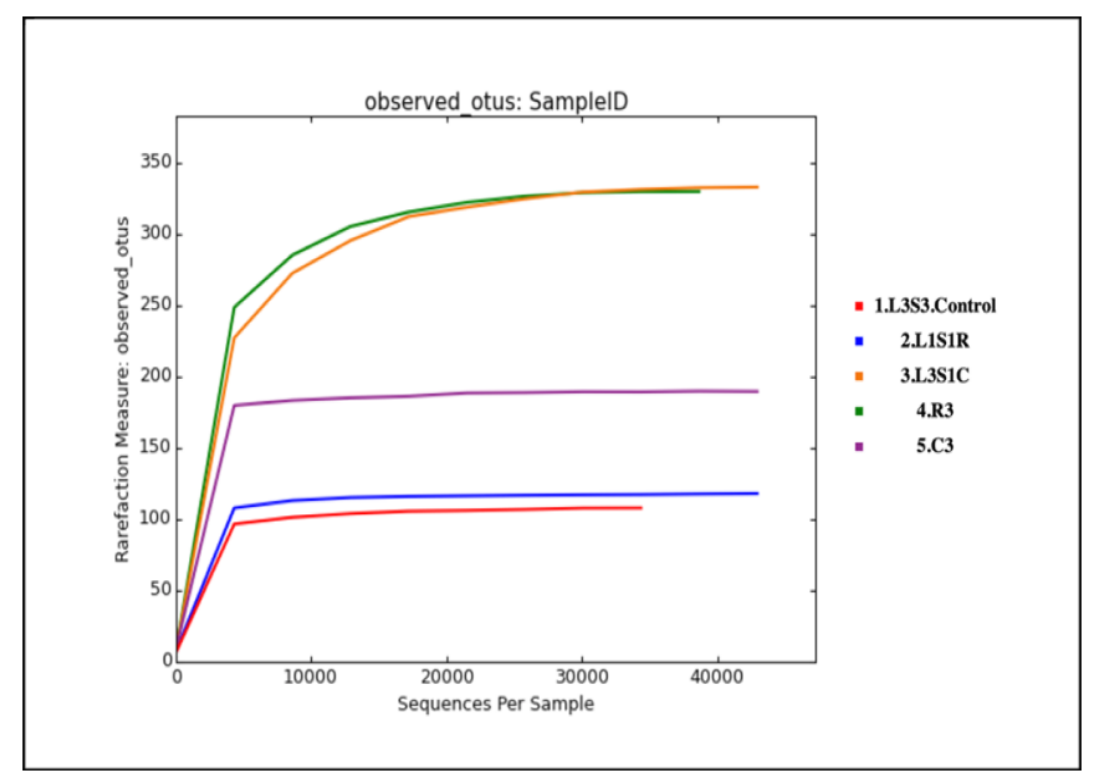

Figure 4. Alfa rarefaction curve observed based on observed species (OTUs) value. (L3. S3

Control): Control sample; (L1.S1.R): H. perfoliata rhizosphere sample; (L1.S1.C): H. perfoliata crust sample; (R3) Z. album rhizosphere sample; (C3) Zygophyllum album crust sample

Principal coordinate analysis (PCoA) was used in order to show the diversity and differences of OTU composition in the different soil samples. PCoA based on OTU abundance of different samples and the unweighted UniFrac that refers to unique species is shown in Figure 5. The red triangle indicates to the control sample. The blue square indicates to the rhizosphere of Halopeplis perfoliata. The orange triangle indicates to the crust of Halopeplis perfoliata. The green circle indicates to the rhizosphere of Zygophyllum album. The Purple triangle indicates to the crust of Zygophyllum album. Similarity is high between samples when they are closely located. 
Table 2. Community richness and diversity

\begin{tabular}{c|c|c|c|c}
\hline \multicolumn{5}{c}{ Community richness and diversity } \\
\hline Sample name & OTUs & Chao1 & Shannon & Inverse Simpson \\
\hline L3S3.Control & 108 & 111 & 4.739486514 & 0.923819556 \\
L1S1R & 118 & 118 & 5.135939679 & 0.939106837 \\
L3S1C & 333 & 343 & 5.799493094 & 0.958314098 \\
R3 & 330 & 330 & 6.429642998 & 0.978116633 \\
C3 & 190 & 190 & 6.747126231 & 0.985840649 \\
\hline
\end{tabular}

Chao1: Species richness estimators estimating the total number of species present in a community by using the frequency of occurrence of rarer OTUs. If a sample contains many singletons or doubletons, it is likely that more undetected OTUs exist, and the Chao 1 index will estimate greater species richness than it would for a sample without rare OTUs

Shannon: A quantitative measure that reflects the number of different types (species) present within a dataset. It also simultaneously takes into account of how evenly the basic entities (individuals) are distributed among those types

Inversed Simpson: An indication of how evenly the species are distributed and measures the degree of concentration when individuals are classified into species

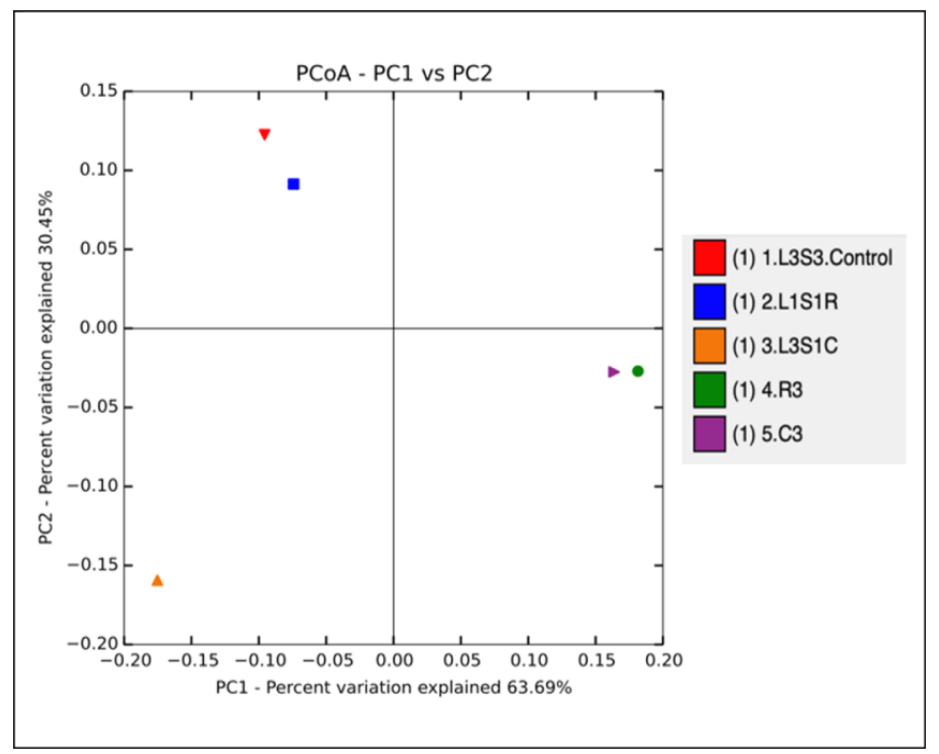

Figure 5. Beta diversity analysis. Unweighted PCoA of UniFrac distances. (L3. S3 Control): Control sample; (L1.S1.R): H. perfoliata rhizosphere sample; (L1.S1.C): H. perfoliata crust sample; (R3) Z. album rhizosphere sample; (C3) Zygophyllum album crust sample

\section{Taxonomic classification at the phyla and genera levels}

Phylogenetic tree based on $16 \mathrm{~S}$ rRNA showing the diversity and taxonomy of bacteria isolated from the five different soil samples at both the phyla and genera levels are shown in (Fig. 6). A phylogenetic tree is a diverging schema presenting the inferred evolutionary relationships among diverse biological taxa constructed upon similarities and differences in their physical or genetic features. The shorter the length of the branch, the closed evolution distance between taxa. Moreover, phylogenetic tree can explain the species evolution relationship in addition to the taxa composition and abundance analysis. 


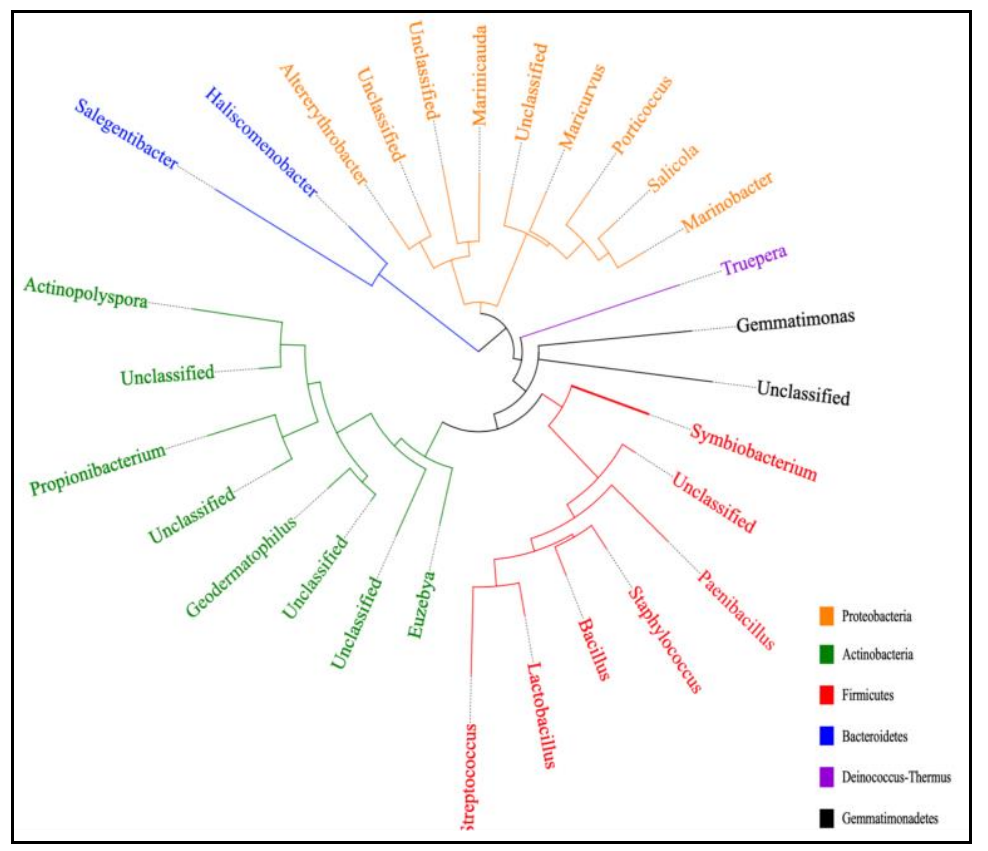

Figure 6. Un-rooted phylogenetic tree based on $16 S$ rRNA gene sequence representing the diversity of bacteria isolated from the various soil samples at booth the phylum and genus level

Results of the bacterial communities in the five soil samples at phylum-level taxonomic distribution showed that they are belongs to nineteen phyla, and the most abundant of them were highlighted for further analysis. The results indicated seven most common phyla which are Proteobacteria (199 genera), Actinobacteria (47 genera), Firmicutes (39 genera), Bacteroidetes (33 genera), Deinococcus-Thermus (one genus), Gemmatimonadetes (one genus), and unclassified phyla (one genus).

Analysis of the bacterial communalities at the phylum classification showed that Proteobacteria and Bacteroidetes were the most found in the sample collected from the crust of the H. perfoliata (3.L3.S1.C) (Fig. 7A, B). Actinobacteria and Firmicutes were significantly showed in the samples collected from both the rhizosphere and the crust of the Zygophyllum album (4.R.3 and 5.C.3) (Fig. 7C, D). Gemmatimonadetes were found in the control sample and the rhizosphere of the H. perfoliata sample (Fig. 7E). Deinococcus-Thermus was found in the control sample followed by the rhizosphere of the H. perfoliata sample (Fig. $7 F$ ). However, the unclassified phyla were found in all of soil samples with a significant abundance in the control sample and the rhizosphere of the $H$. perfoliata sample (Fig. $7 G$ ).

The previously mentioned highly abundant seven bacteria found at the phylum-level include large numbers of genera, estimated at 321 genera. At the genus level, Actinopolyspora, Geodermatophilus, Propionibacterium, Euzebya, and four unclassified were the most abundant found in the Actinobacteria. Salegentibacter, Haliscomenobacter, and one unclassified genus were found in Bacteroidetes. Whereas Sphaerobacter and one genus unclassified were found in Chloroflexi. Truepera was found in Deinococcus-Thermus, and Gemmatimonas was found in Gemmatimonadetes. Marinicauda, Altererythrobacter, Maricurvus, Marinobacter, Porticoccus, Salicola, and three unclassified were found in Proteobacteria. Bacillus, Staphylococcus, Paenibacillus, Lactobacillus, Streptococcus, Symbiobacterium, and one unclassified genus were found in Firmicutes (Fig. 8). 


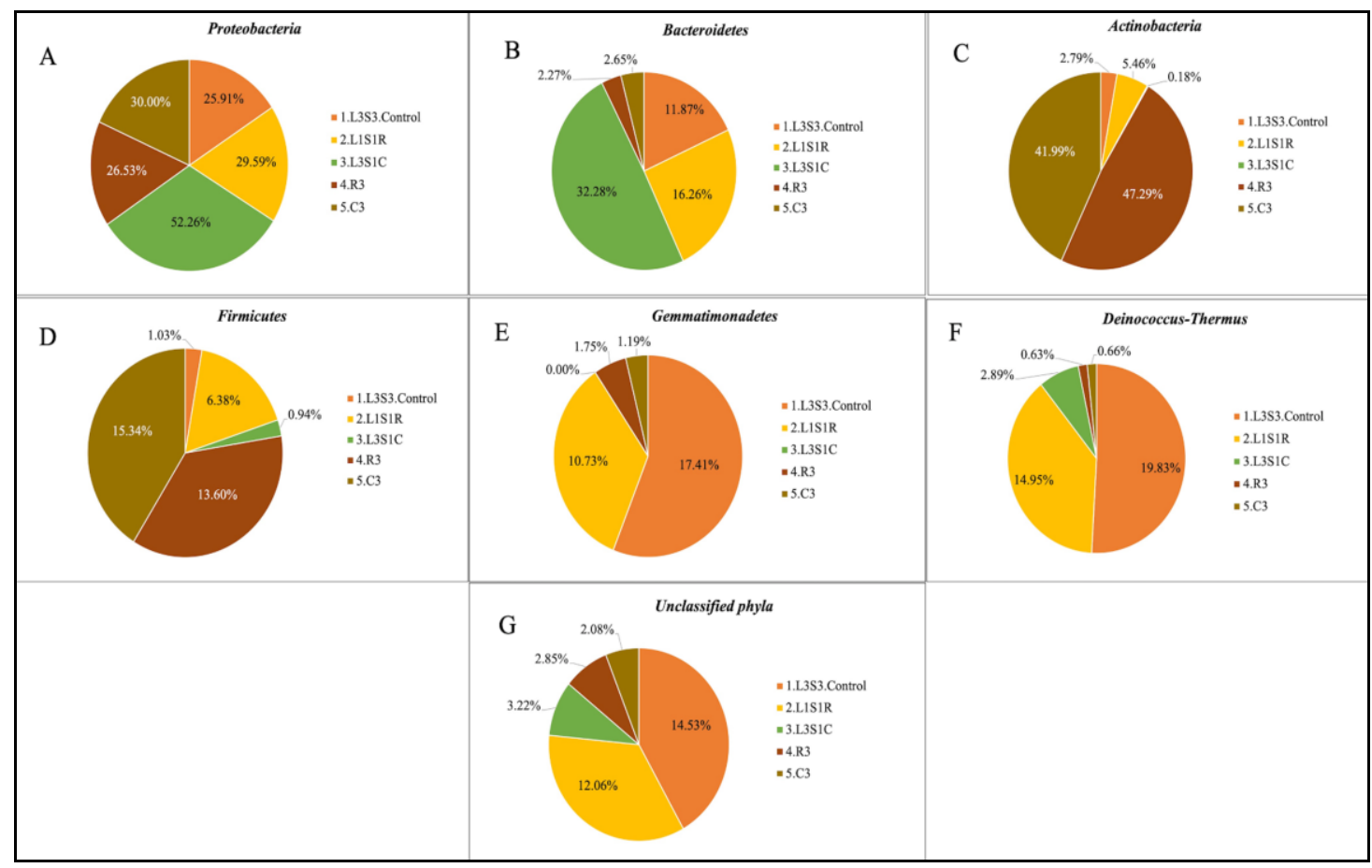

Figure 7. bacterial communities at the phylum classification among the samples. (A) The amount of Proteobacteria among the samples. (B) The amount of Bacteroidetes among the samples. (C) The amount of Actinobacteria among the samples. (D) The amount of Firmicutes among the samples. $(E)$ The amount of Gemmatimonadetes among the samples. $(F)$ The amount of Deinococcus-Thermus among the samples. $(G)$ The amount of the unclassified phyla among the samples. (L3. S3 Control): Control sample; (L1.S1.R): H. perfoliata rhizosphere sample; (L1.S1.C): H. perfoliata crust sample; (R3) Z. album rhizosphere sample; (C3) Zygophyllum album crust sample

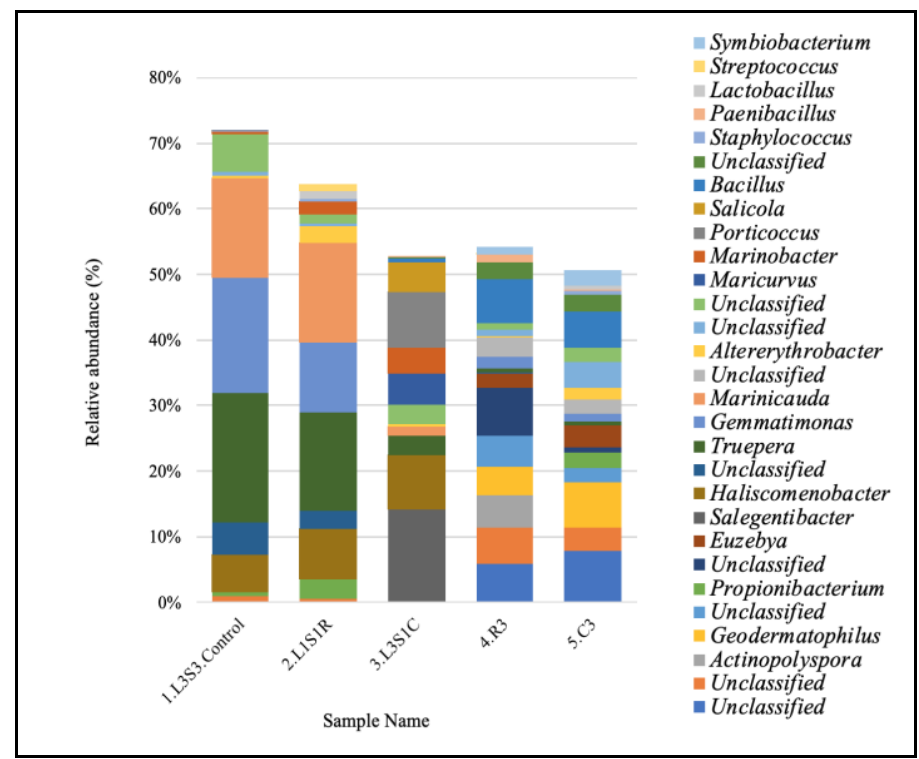

Figure 8. The relative most abundance in the taxonomic composition distribution in samples of Genus -level. (L3. S3 Control): Control sample; (L1.S1.R): H. perfoliata rhizosphere sample; (L1.S1.C): H. perfoliata crust sample; (R3) Z. album rhizosphere sample; (C3) Zygophyllum album crust sample 


\section{Discussion}

Over the past decade, the interest in studying the bacterial diversity in soil salinity and the hot desert has increased due to global climate change with arid regions believed to be more vulnerable (Osman et al., 2016). The purpose of the presented study was to discover the bacterial diversity that inhabit salty soil associated with halophytic plants, which located in the Southern Corniche of Jeddah, Saudi Arabia, in order to pinpoint beneficial strains that promote crops to survive with different environmental stress and have several industrials outcomes. Bacterial cultivationbased isolation methods, which are commonly used, are not very effective due to the limitation of several factors including media compositions, nutritional and environmental requirements of soil microbial communities (Fierer et al., 2012). Our microbiome analysis involves sampling collection, processing, NGS sequencing, and bioinformatics analysis to provide composition of those microbiota populations associated with halophytes. Five various soil samples were collected from different locations of the same area which included; the rhizosphere and crust of both the $H$. perfoliata and the Z. album plants. The fifth sample was collected from an area that has no plant and was considered as a control. Amplification of the v1-v3 bacterial 16S rRNA genes regions by PCR detected bacterial biodiversity in these extreme conditions. Therefore, 546,713 high-quality sequences were obtained and classified at the phylum and genus levels, and the differences between bacterial combinations were studied in the five soil samples. The richness and diversity of the bacteria were examined in each sample, and a slight change was found among the samples (from 108 to 333 OTUs) in the five samples from the same region.

Sequencing results showed that the taxonomic distribution of the bacterial communities at the phylum-level indicated nineteen phyla. More importantly, numerous studies have shown that these bacteria have many environmental and ecological benefits including plant growth-promoting bacteria (PGPB) (Gupta et al., 2015). The most dominant phyla among the five different soil samples were Proteobacteria. Proteobacteria have been used as a biological treatment for various toxic complexes and as naturally bioactive products (Bodenhausen et al., 2013; Mukhtar et al., 2018). Moreover, they are well known to be sensitive to climate change and have a influence on the soil biosphere due to their involvement in the global carbon, nitrogen and sulfur cycles (Zhao et al., 2018). Actinobacteria were found frequently in the rhizosphere and the crust of the $Z$. album samples. Actinobacteria are commonly used as a source of active antimicrobial biomaterials (Elbendary et al., 2018). They are also essential in restating the uncontrolled biomaterials through the decomposition of plants and dead animals. Moreover, they have a significant function in the production of antibiotics and providing the high resistance of the UV radiation and dehydration (Barka et al., 2016; Zhao et al., 2018). Bacteroidetes were shown to be a sensitive biological indicator for agricultural soil use and they have a potential association between antibacterial and antifungal performance (Eida et al., 2018; Wolińska et al., 2017). Firmicutes were found to be more abundance in both of the Z. album samples. They have been used as a prevailing species among all the marine enzyme-producing microscopic organisms (Divya et al., 2010). They are able to produce salt stress compounds to overcome salt causing osmotic pressures (Meena et al., 2017). Gemmatimonadetes were found to be more in the control and the rhizosphere of the H. perfoliata sample. They are able to live under aerobic and anaerobic respiration, and this indicates that they can adapt low soil 
moisture (DeBruyn et al., 2011). They also play a major role in the biochemical transformations (Kadam and Chuan, 2016; Zhang et al., 2003). Deinococcus-Thermus were found to be significant in the control sample in addition to both of the $H$. perfoliata sample and they were known to be highly resistant to harsh environmental stresses and radiation (Theodorakopoulos et al., 2013). Unclassified bacteria at the phylum level were found with $2.08 \%$ in the crust of the Z. album sample and $14.53 \%$ in the control sample. The emergence of the unclassified bacteria at the phylum-level may be due to the lack of a reference sequence in the database and theses bacteria might include a potential candidate that are still not identified.

At the genus-level, several studies have shown the benefits of the bacteria in agricultural, environmental, medical or industrial applications. For example, Altererythrobacter, one of the most abundant genera were found in the soil samples, which fall under the Proteobacteria. Many physiological studies have notified that Altererythrobacter strains possess degrading activity against rebellious organic petroleum-derived hydrocarbons (Maeda et al., 2018). Propionibacterium (of Actinobacteria Phylum) one of the genera that were found in the soil sample and thy are widely used in many applications including the production of vitamin B12 and probiotic and cheese industries (Kiatpapan and Murooka, 2002). Bacillus were found significantly and they are well known as a source of antibiotics and probiotics. There are a few types of Bacillus that hurtful to human Bacillus anthracis which cause anthrax and Bacillus cereus that are responsible for the food poisoning. Moreover, some strains have a greater capacity not only in the soil but also in their ability to produce compounds that can be used for several applications (Ahmad et al., 2018). Bacillus subtilis and Bacillus halotolerans were shown to produce Polyhydroxyalkanoates (PHAs) that were found to be used as an alternative for petrochemical-derived plastic (Valappil et al., 2007). PHAs were found to be ecofriendly, biodegradable, biocompatible and microbial thermoplastic (El-Hamshary et al., 2018; Zaki, 2018).

\section{Conclusion}

This study shows diversity of the microbial communities that present in the soil associated with stress tolerance halophytic plants located in the Southern Corniche of Jeddah, Saudi Arabia. The Kingdom of Saudi Arabia is characterized by extraordinary environments beginning from the brutal desert of the Arabian Peninsula, passing through the salty areas and saline marshes and ending with the Red Sea which is known by its exceptional decent variety. Microbial communities inhibited this ecosystem should be investigated. Our finding showed that rhizospheric microbes can be studied as biomarkers of plant growth rate as well as its power to survive under harsh environmental conditions. This will give a better understanding for novel candidates that can be used as biological agents to improve agricultural and industrial practices. In addition to the identification of the soil bacterial communities through high-throughput molecular tools for the characterization of the taxonomic and phylogenetic, future detailed representation and comparative functional and biochemical studies for the diversity of the soil microbiome is needed to highlight different metabolic pathways. Moreover, future correlation between the taxonomic composition and the functional characteristics of the soil microbiome, will help in the discovery of novel promising candidates to improve the fate of humankind and its resources. 
Acknowledgements. This work was funded by the Deanship of Scientific Research (DSR), University of Jeddah, Jeddah, under grant No. (UJ-01-18-ICP). The authors, therefore, acknowledge with thanks DSR technical and financial support.

Conflict of interests. The authors declare that they have no conflict of interests.

\section{REFERENCES}

[1] Ahmad, M., Pataczek, L., Hilger, T. H., Zahir, Z. A., Hussain, A., Rasche, F., Schafleitner, R., Solberg, S. Ø. (2018): Perspectives of microbial inoculation for sustainable development and environmental management. - Frontiers in Microbiology 9: 2992.

[2] Al-Oudat, M., Qadir, M. (2011): The Halophytic flora of Syria. - International Center for Agricultural Research in the Dry Areas (ICARDA) Aleppo, Syria 8: 186.

[3] Aeshen, M. N. (2017): Metagenomics of microbial communities associated with the rhizosphere of the Saudi Desert medicinal plants. - EC Microbiology ECO 1: 31-33.

[4] Barka, E. A., Vatsa, P., Sanchez, L., Gaveau-Vaillant, N., Jacquard, C., Klenk, H. P., Clément, C., Ouhdouch, Y., van Wezel, G. P. (2016): Taxonomy, physiology, and natural products of Actinobacteria. - Microbiology and Molecular Biology Reviews 80: 1-43.

[5] Batanouny, K. H., Baeshin, N. A. (1982): Studies on the flora of Arabia II. The MedinaBadr Road. Saudi Arabia. - Bull. Fac. Sci., KAU, Jeddah 6: 1-26.

[6] Batanouny, K. H., Baeshin, N. A. (1983): Plant communities along the Medina-Badr road across the Hejaz mountains, Saudi Arabia. - Vegetatio 53: 33-43.

[7] Bodenhausen, N., Horton, M. W., Bergelson, J. (2013): Bacterial communities associated with the leaves and the roots of Arabidopsis thaliana. - PLOS One 8.

[8] Bui, E. N. (2013): Soil salinity: a neglected factor in plant ecology and biogeography. Journal of Arid Environments 92: 14-25.

[9] Caporaso, J. G., Kuczynski, J., Stombaugh, J. I., Bittinger, K., Bushman, F. D., Costello, E. K., Fierer, N., Pena, A. G., Goodrich, J. K., Gordon, J. I., Huttley, G. A. (2010): QIIME allows analysis of high-throughput community sequencing data. - Nature Methods 7: 335 .

[10] Chao, A., Bunge, J. (2002): Estimating the number of species in a stochastic abundance model. - Biometrics 58: 531-539.

[11] Chaparro, J. M., Sheflin, A. M., Manter, D. K., Vivanco, J. M. (2012): Manipulating the soil microbiome to increase soil health and plant fertility. - Biology and Fertility of Soils 48: 489-499.

[12] de Zelicourt, A., Al-Yousif, M., Hirt, H. (2013): Rhizosphere microbes as essential partners for plant stress tolerance. - Molecular Plant 6: 242-245.

[13] DeBruyn, J. M., Nixon, L. T., Fawaz, M. N., Johnson, A. M., Radosevich, M. (2011): Global biogeography and quantitative seasonal dynamics of Gemmatimonadetes in soil. Applied and Environmental Microbiology 77: 6295-6300.

[14] del Carmen Orozco-Mosqueda, M., del Carmen Rocha-Granados, M., Glick, B. R., Santoyo, G. (2018): Microbiome engineering to improve biocontrol and plant growthpromoting mechanisms. - Microbiological Research 208: 25-31.

[15] Divya, B., Soumya, K. V., Nair, S. (2010): 16SrRNA and enzymatic diversity of culturable bacteria from the sediments of oxygen minimum zone in the Arabian Sea. Antonie van Leeuwenhoek 98: 9-18.

[16] Eida, A. A., Ziegler, M., Lafi, F. F., Michell, C. T., Voolstra, C. R., Hirt, H., Saad, M. M. (2018): Desert plant bacteria reveal host influence and beneficial plant growth properties. - PLOS One 13. 
[17] El Ghoul, J., Ghanem-Boughanmi, N., Ben-Attia, M. (2011): Biochemical study on the protective effect of ethanolic extract of Zygophyllum album on streptozotocin-induced oxidative stress and toxicity in mice. - Biomedicine \& Preventive Nutrition 1: 79-83.

[18] El-Hamshary, O. I. M., Kadi, H. A., Al-Twaty, N. H. (2018): Molecular characterization and UV improvement of some bioplastic-producing bacteria isolated from plants in Taif City, Saudi Arabia. - Pharmacophore 9.

[19] Elbendary, A. A., Hessain, A. M., El-Hariri, M. D., Seida, A. A., Moussa, I. M., Mubarak, A. S., Kabli, S. A., Hemeg, H. A., El Jakee, J. K. (2018): Isolation of antimicrobial producing actinobacteria from soil samples. - Saudi Journal of Biological Sciences 25: 44-46.

[20] Etesami, H., Beattie, G. A. (2018): Mining halophytes for plant growth-promoting halotolerant bacteria to enhance the salinity tolerance of non-halophytic crops. - Frontiers in Microbiology 9: 148.

[21] Fierer, N., Leff, J. W., Adams, B. J., Nielsen, U. N., Bates, S. T., Lauber, C. L., Owens, S., Gilbert, J. A., Wall, D. H., Caporaso, J. G. (2012): Cross-biome metagenomic analyses of soil microbial communities and their functional attributes. - Proceedings of the National Academy of Sciences 109: 21390-21395.

[22] Glick, B. R. (2012): Plant growth-promoting bacteria: mechanisms and applications. Scientifica 2012: 15 .

[23] Gupta, G., Parihar, S. S., Ahirwar, N. K., Snehi, S. K., Singh, V. (2015): Plant growth promoting rhizobacteria (PGPR): current and future prospects for development of sustainable agriculture. - Journal of Microbial and Biochemical Technology 7: 96-102.

[24] Kadam, P. D., Chuan, H. H. (2016): Erratum to: Rectocutaneous fistula with transmigration of the suture: a rare delayed complication of vault fixation with the sacrospinous ligament. - International Urogynecology Journal 27: 505-505.

[25] Kiatpapan, P., Murooka, Y. (2002): Genetic manipulation system in propionibacteria. Journal of Bioscience and Bioengineering 93: 1-8.

[26] Maeda, A. H., Nishi, S., Ishii, S. I., Shimane, Y., Kobayashi, H., Ichikawa, J., Kurosawa, K., Arai, W., Takami, H., Ohta, Y. (2018): Complete genome sequence of Altererythrobacter sp. Strain B11, an Aromatic monomer-degrading bacterium, isolated from deep-sea sediment under the seabed off Kashima, Japan. - Genome Announcements 6: e00200-18.

[27] Majeed, M. A., Baeshen, M. N., Afifi, M., Ahmed, F. (2020): Metagenomics of bacterial communities associated with the halophytic plant. - EC Microbiology 16: 1-11.

[28] Meena, K. K., Sorty, A. M., Bitla, U. M., Choudhary, K., Gupta, P., Pareek, A., Singh, D. P., Prabha, R., Sahu, P. K., Gupta, V. K. a., Singh, H. B. (2017): Abiotic stress responses and microbe-mediated mitigation in plants: the omics strategies. - Frontiers in Plant Science 8: 172.

[29] Mnafgui, K., Hamden, K., Ben Salah, H., Kchaou, M., Nasri, M., Slama, S., Derbali, F., Allouche, N., Elfeki, A. (2012): Inhibitory activities of Zygophyllum album: a natural weight-lowering plant on key enzymes in high-fat diet-fed rats. - Evidence-Based Complementary and Alternative Medicine. https://doi.org/10.1155/2012/620384.

[30] Mukhtar, S., Mirza, B. S., Mehnaz, S., Mirza, M. S., Mclean, J., Malik, K. A. (2018): Impact of soil salinity on the microbial structure of halophyte rhizosphere microbiome. World Journal of Microbiology and Biotechnology 34: 136.

[31] Osman, J. R., Zelicourt, A. D., Bisseling, T., Geurts, R., Hirt, H., DuBow, M. S. (2016): Bacterial rhizosphere biodiversity from several pioneer desert sand plants Near Jizan, Saudi Arabia. - The Open Conference Proceedings 7: 70-79.

[32] Rasool, S. G., Hameed, A., Khan, M. A., Gul, B. (2017): Seeds of Halopeplis perfoliata display plastic responses to various abiotic factors during germination. - Flora 236-237: 76-83.

[33] Rawat, N., Joshi, G. K. (2019): Bacterial community structure analysis of a hot spring soil by next generation sequencing of ribosomal RNA. - Genomics 111: 1053-1058. 
[34] Sejiny, M. J., Zaki, M. M., Hamed, A. S., Baeshin, N. A. (1980): Psychrophilic and mesophilic microflora in soil and rhizosphere of some Juniperus forest plants at Al-Shafa region, Taif, Saudi Arabia. - Bull. Fac. Sci. KAU 4: 109-116.

[35] Theodorakopoulos, N., Bachar, D., Christen, R., Alain, K., Chapon, V. (2013): Exploration of Deinococcus-Thermus molecular diversity by novel group-specific PCR primers. - Microbiology Open 2: 862-872.

[36] Tigrine-Kordjani, N., Youcef Meklatib, B., Chemat, F. (2011): Contribution of microwave accelerated distillation in the extraction of the essential oil of Zygophyllum album L. - Phytochemical Analysis 22: 1-9.

[37] Timmusk, S., Behers, L., Muthoni, J., Muraya, A., Aronsson, A. C. (2017): Perspectives and challenges of microbial application for crop improvement. - Frontiers in Plant Science 8: 49.

[38] Valappil, S. P., Peiris, D., Langley, G. J., Herniman, J. M., Boccaccini, A. R., Bucke, C., Roy, I. (2007): Polyhydroxyalkanoate (PHA) biosynthesis from structurally unrelated carbon sources by a newly characterized Bacillus spp. - Journal of Biotechnology 127: 475-487.

[39] Waśkiewicz, A., Beszterda, M., Goliński, P. 2013. ABA: Role in Plant Signaling Under Salt Stress. - In: Ahmad, P. et al. (eds.) Salt Stress in Plants. Springer, New York.

[40] Wolińska, A., Kuźniar, A., Zielenkiewicz, U., Izak, D., Szafranek-Nakonieczna, A., Banach, A., Błaszczyk, M. (2017): Bacteroidetes as a sensitive biological indicator of agricultural soil usage revealed by a culture-independent approach. - Applied Soil Ecology 119: 128-137.

[41] Zaki, M. M., Hamed, A. S., Sejiny, M. J., Baeshin, N. A., Younes, H. A. (1980): Halophilic bacteria in soil and rhizosphere of some littoral salt marsh plants at Shuaiba Lagoon, Saudi Arabia. - Bull. Fac. Sci. KAU 4: 91-100.

[42] Zaki, N. H. (2018): Biodegradable plastic production by Bacillus spp. isolated from agricultural wastes and genetic determination of PHA synthesis. - Al-Mustansiriyah Journal of Science 29: 67-74.

[43] Zhang, H., Sekiguchi, Y., Hanada, S., Hugenholtz, P., Kim, H., Kamagata, Y., Nakamura, K. (2003): Gemmatimonas aurantiaca gen. nov., sp. nov., a Gram-negative, aerobic, polyphosphate-accumulating micro-organism, the first cultured representative of the new bacterial phylum Gemmatimonadetes phyl. nov. - International Journal of Systematic and Evolutionary Microbiology 53: 1155-1163.

[44] Zhao, Y., Song, C., Dong, H., Luo, Y., Wei, Y., Gao, J., Wu, Q., Huang, Y., An, L., Sheng, H. (2018): Community structure and distribution of culturable bacteria in soil along an altitudinal gradient of Tianshan Mountains, China. - Biotechnology \& Biotechnological Equipment 32: 397-407.

[45] Zreik, R. (1990): The domestication and economic cultivation of halophytes. Developing World Agriculture 8: 74-79. 


\section{APPENDIX}

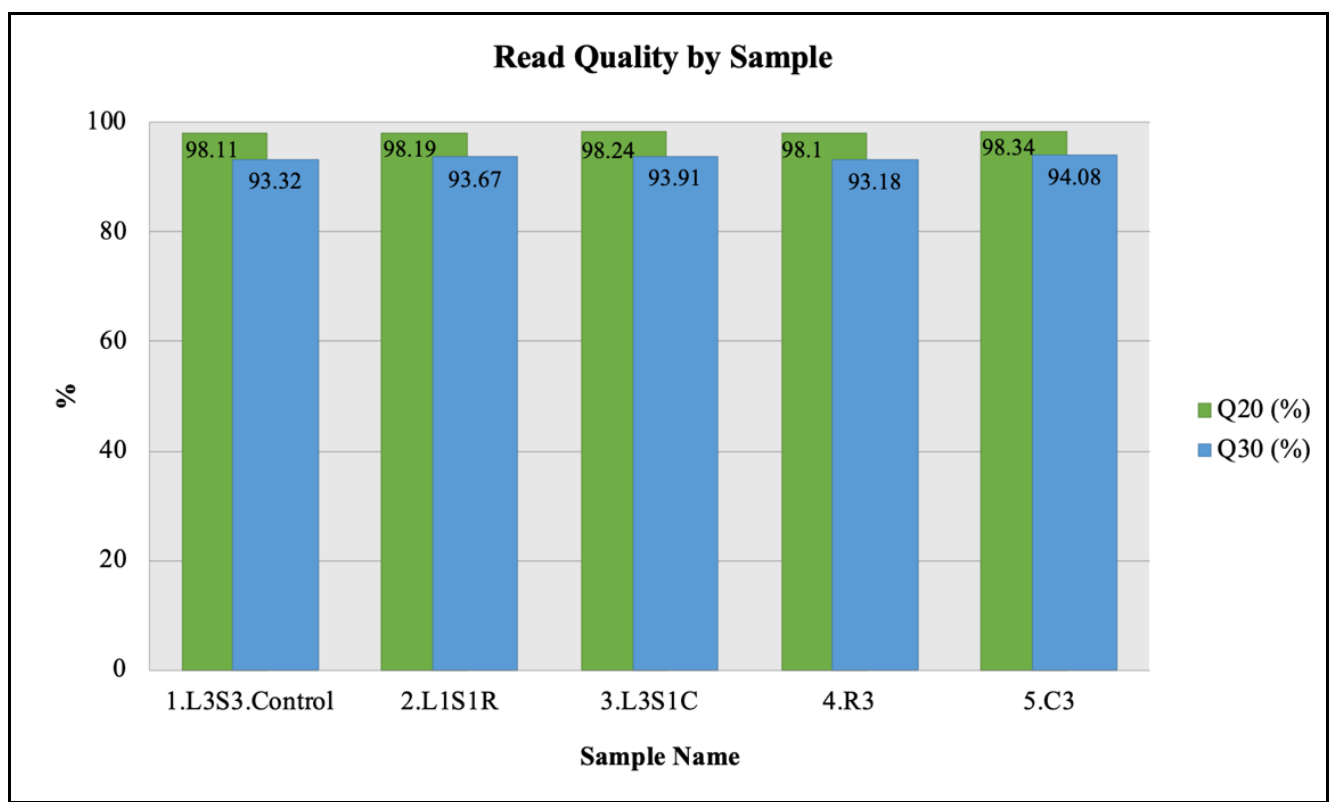

Figure A1. The percentage of read quality of the five soil different samples. Q20(\%): The percentage of bases in which the phred score is above 20. Q30(\%): The percentage of bases in which the phred score is above 30. (L3. S3 Control): Control sample; (L1.S1.R): H. perfoliata rhizosphere sample; (L1.S1.C): H. perfoliata crust sample; (R3) Z. album rhizosphere sample; (C3) Zygophyllum album crust sample

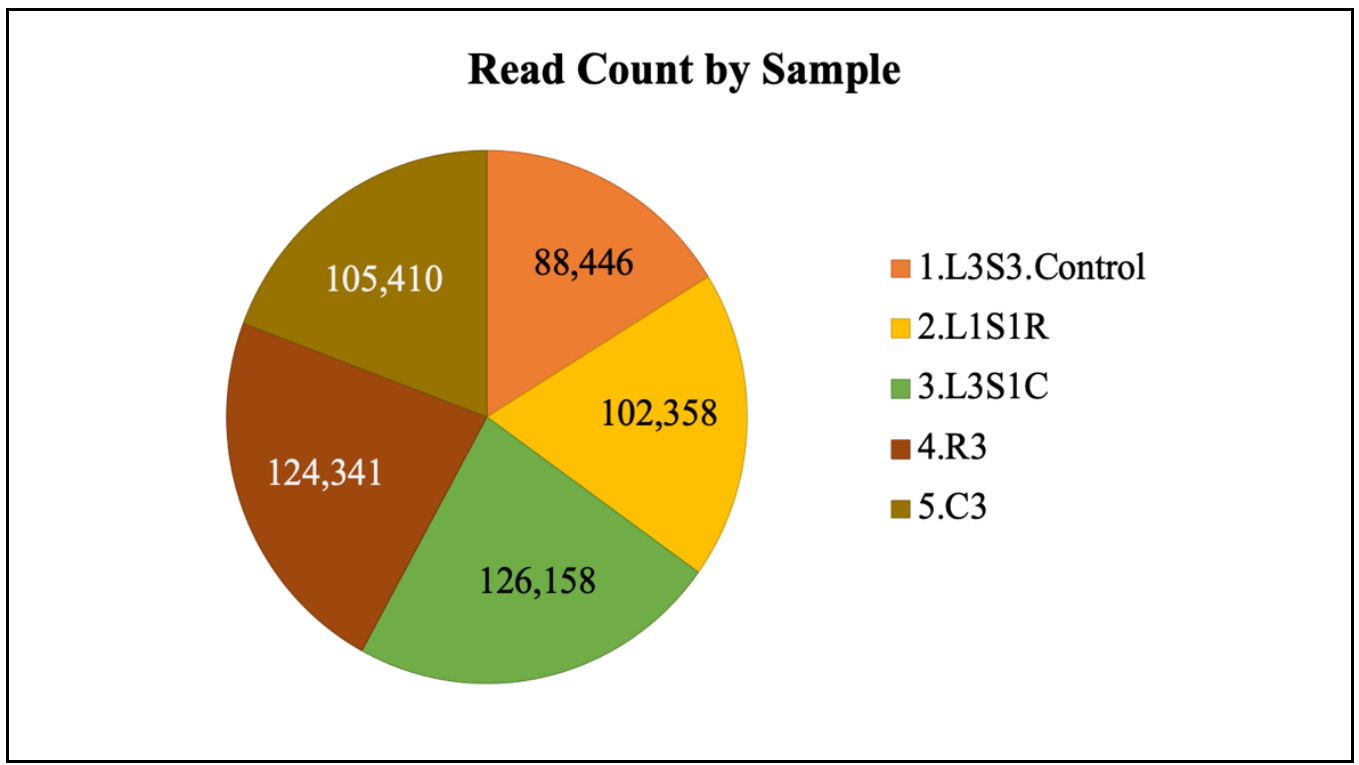

Figure A2. The total number of sequences reads among the five soil samples. (L3. S3 Control): Control sample; (L1.S1.R): H. perfoliata rhizosphere sample; (L1.S1.C): H. perfoliata crust sample; (R3) Z. album rhizosphere sample; (C3) Zygophyllum album crust sample 